\title{
XLIII. An attempt towards a theory of the resistance experienced by two and four-wheeled carriages on different kinds of roads; and to determine the circumstances under which the one are preferable to the other
}

\section{Nicholas Fuss}

To cite this article: Nicholas Fuss (1802) XLIII. An attempt towards a theory of the resistance experienced by two and four-wheeled carriages on different kinds of roads; and to determine the circumstances under which the one are preferable to the other , Philosophical Magazine Series 1, 13:51, 257-266, DOI: 10.1080/14786440208676124

To link to this article: http://dx.doi.org/10.1080/14786440208676124

\section{Published online: 18 May 2009.}

\section{Submit your article to this journal $\longleftarrow$}

\section{Џll Article views: 2}


For good figures of the C. cancellatus, Micheli may be referred to, and for the two latter, Curtis's FI. Lond. and Sowerby's Englifh Fungi, called in both publications PHa Lus.

I am not aware that any other Fungus will arrange with the above three fpecies, although fome have been placed under the genus PHaluis, by Linnæus and other botanifts, which have very little agreenent with the P. impudicus and P. caninus of thole authors.

XLIII. An Attempt towards a Theory of tbe Refiftance experienced by trvo and four-wbeeled Carriages on differcnt Kinds. of Roads; and to ditermine the Circumftances under wobicls the one are preferable to the olber. By Nichol as Fuss, Profeffor of the biglber Matbematiss at Peterfburgh, Member of the Inperial Acudeny of Sciences, \&o.

[Continued from p. 122.]

Second Division.

Of the Refiftance on folid and unerven Roads.

I. Four-avheded Carriages.

Section I9.

$\mathrm{I}_{\mathrm{F}}$ F the road $A B$, Plate II. fig. 4. be naturally rough and ftony, or paved artificially, or covered in fome places with trees laid acrols it, new inpediments arife from thefe inequalities, and the refiftance found in the preceding divifion, fection 9, which takes place here alfo, acquires an increafe, which is determined in the following manner:

Let the wheel touch in $G$ and $G^{\prime}$ two equally large and folid inequalities of the road, and for the fore-wheels let the angle $\mathrm{GOG}^{\prime}=2 \phi$, but for the hind-wheels $\mathrm{GOG}^{\prime}=2 \psi$. Now as a part of the moving power OV, which we thall call $\mathrm{OU}=K$, muft be employed to overcome a part of the power of gravity acting in a direction perpendicular to $A B$, which is $\left({ }_{2}^{1} \mathrm{P}+p\right) \cos . \alpha$, and to raife the fore-axle together with its part of the load over the fixed point $G$, the momentum of thefe powers OU.GU and OR.GR muft be equal to each other; that is, K.GO cos. $\phi=\left(\frac{1}{2} P+p\right) \cos . \alpha . G O$ tin. $\phi$; fo that the increate of refiftance arifing from this impediment will be for the fore-wheels $\mathrm{K}=\left(\frac{1}{2} \mathrm{P}+p\right) \cos \alpha$ tang. $\varphi . \quad \mathrm{A}$ fimilar increafe will be found for the hind-wheels $=\left(\frac{1}{2} P+p^{\prime}\right)$ cos. $\alpha$ tang. $\psi$. Confcquently the whole refiftance on roads of the fecond clafs for four-wheeled carriages is : $\left.\begin{array}{rl}R & =\frac{1}{2}(m+n) \lambda P \cos a+\left(P+p+p^{\prime}\right) \text { fin. } \alpha \\ & +\left(\frac{1}{2} P+p\right) \cos \alpha \text { tang. } \phi+\left(\frac{1}{2} P+p^{\prime}\right) \cos . \alpha \text { tang. } \psi\end{array}\right\}$ 


\section{Two-wubeled Carriages. \\ Seciion 20.}

The refiftance found in the preceding divifion, fection 12, for carriages of this kind, acquires here an increafe, which, if we make the angle $\mathrm{GOG}^{\prime}=2 \omega$, will be found in the fame manner as in the preceding fection $=(\mathrm{P}+\pi) \cos . \alpha$ tang. $\omega_{\text {. }}$ The whole refftance for two-wheeled carriages on roads of the fecond clafs will therefore be expreffed as follows :

$$
\left.\begin{array}{rl}
R^{\prime}= & \mu \lambda P \cos , \alpha+(P+\varepsilon P+\pi) \text { fin. } \alpha \\
& +(P+\pi) \cos . \alpha \text { tang. } \omega
\end{array}\right\}
$$

III. Comparijon of the two Kinds of Carriages.

Section $2 \mathrm{x}$.

If the comparifon be made in general according to the five different points of view mentioned in the 16 th fection, it will be found that four-wheeled carriages are preferable to twowheeled when any of the five following conditions take place:

$$
\text { Ift, } \left.\begin{array}{rl}
\frac{m+n-2 \mu}{2} & \frac{\left(\varepsilon \mathrm{P}+\pi-p-p^{\prime} \tan \cdot \alpha+(\mathrm{P}+\pi) \tan \omega\right.}{\lambda \mathrm{P}} \\
\frac{-\left(\frac{\mathrm{T}}{2} \mathrm{P}+p\right) \operatorname{tang} \cdot \phi-\left(\frac{1}{2} \mathrm{P}+p^{7}\right) \operatorname{tang} \cdot \psi}{\lambda \mathrm{P}}
\end{array}\right\}
$$

$$
\begin{aligned}
& \left.2 \mathrm{~d}, p+p^{\prime}-\pi<\varepsilon \mathrm{P}-\frac{(m+n-2 \mu) \frac{1}{2} \lambda \mathrm{P}+(\mathrm{P}+\pi) \text { tang. } \omega}{\operatorname{tang} \alpha}\right\} \\
& \frac{-\left(\frac{1}{2} P+p\right) \text { tang. } \phi-\left(\frac{1}{2} P+p^{\prime}\right) \operatorname{tang} \cdot \psi}{\operatorname{tang} \alpha}
\end{aligned}
$$

$3^{\text {d, }} \mathrm{P}>\frac{\left(p+p^{\prime}-\pi\right) \text { tang. } \alpha+p \text { tang. } \phi+p^{\prime} \text { tang. } \psi-\pi \text { tang. } \omega}{\varepsilon \text { tang. } \alpha+\text { tang, } \omega-\frac{7}{2} \operatorname{tang} \phi-\frac{3}{2} \psi-\frac{1}{2} \lambda(m+n-2 \mu)}$

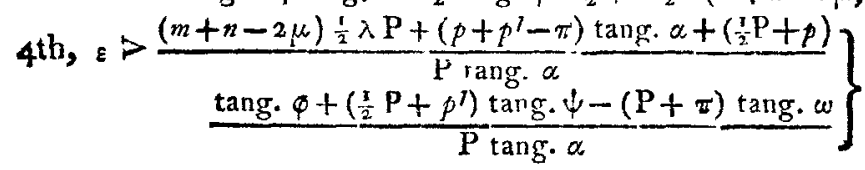

$$
\text { 5th, Tang, } \left.a>\frac{(m+n-2 \mu) \frac{1}{2} \lambda \mathrm{P}+\left(\frac{1}{2} \mathrm{P}+p\right) \text { tang. } \phi}{\varepsilon \mathrm{P}+\pi-p-p^{\prime}}\right\}
$$

EXAMPLE 1.

\section{ScEzion 22.}

Let the road be compofed of round pieces of timber fix inches in diameter laid acrofs it, and let $a=0$. Let the mean diameter of the fore and hind axle-trees of the fourwheeled carriages be $3 \frac{x}{4}$ inches; that of the axles of the twowheeled carriages $3 \frac{3}{4} ;$ and the diameter of the wheels in the 
fame order, 26, 39, and 45 inches; fo that $m=\frac{x}{8}, n=\frac{x}{12}$, and $\mu=\frac{1}{T^{2}}$ : allo, let $P=1000$ lib., $p=74$ lib., $p^{\prime}=106$ lib., and $\pi=130$ lib.; and let the coefficient of the friction be $\lambda$ $=\frac{2}{9}$. In the laft place, as TG $=\mathrm{TF}=3$ inches, we thall have for the wheels in the above order TO $=16, \frac{45}{2}$ and $\frac{5 x}{2}$ inches, and becaufe fin. TOF $=\frac{T F}{T O}$; the angle TOF, that is to fay, $\varphi=10^{\circ} 48^{\prime} ; \psi=7^{\circ} 40^{\prime} ; \omega=6^{\circ} 45^{\circ}$. From thefe elements we obtain,

$$
\begin{aligned}
\frac{x}{2}(m+n) \lambda \mathrm{P} & =23.148 \\
\left(\frac{1}{2} \mathrm{P}+p\right) \text { tang. } \phi & =109.496 \\
\left(\frac{1}{2} \mathrm{P}+p\right) \text { tang. } \psi & =81.575 \\
\mathrm{R} & =214^{\circ} \cdot 19 \\
& =18.518 \\
\mu \lambda \mathrm{P} & =133.744 \\
(\mathrm{P}+\pi) \text { tang. } \omega & =152.262
\end{aligned}
$$

For a horfe, then, whofe ftrength $\mathrm{M}=400 \mathrm{lib}$. and velocity $\mathrm{G}=13$ feet on fuch a road, we have $g=3 \cdot 484$ feet, and $g^{\prime}=4^{\circ} 979$ feet in a fecond.

It might be conjectured that four-wheeled carriages, the fore-wheels being fo fmall, would experience a refiftance confiderably greater than the two-wheeled. Were $m=n$ $=\frac{x}{12}$, and $p=p^{\prime}=106$, we thould have $\mathrm{R}=181.669$, and $g=4 \cdot 238$.

\section{EXAMPLE II.}

\section{Section 23.}

Let the road be fteep and compofed of ftones, the angle of elevation being $\alpha=14^{\circ}$, and let the pavement be of fuch a nature, that the diftance between the points of contact $G$, $\mathrm{G}^{\prime}=3$ inches, confequently the angle $\phi=5^{\circ} 45^{\circ} ; \psi=4^{\circ} 6^{\prime}$, and $\omega=3^{\circ} 49^{\prime}$. Let the burden be $P=1800$ lib., the mean diameter of the axle-trees of the fore-wheels $=3 \frac{1}{3}$ inches, of the hind-wheels $3 \frac{1}{2}$ inches, that of the two-wheeled carriages 3 inches; let the diameter of the wheels in the fame order be 30,42 , and 45 inches; fo that $m=\frac{1}{2}, n=\frac{1}{2}$, and $\mu=\frac{1}{2}$. The weight of the wheels we hall fuppofe to be $p=80$ lib. $p^{\prime}=110$ lib., and $\pi=120$ lib. In the laft place, let $\lambda=\frac{x}{5}$, and $\varepsilon=\frac{1}{2+}$. Hence we obtain

$$
\begin{aligned}
\frac{1}{2}(m+n) \lambda \mathrm{P} \cos . \alpha & =33.960 \\
\left(\mathrm{P}+p+p^{\prime}\right) \text { fin. } \alpha & =48 \mathrm{1} \cdot 425 \\
\left(\frac{\mathrm{L}}{2} \mathrm{P}+p\right) \cos . \alpha \text { tang. } \phi & =95.750 \\
\left(\frac{\mathrm{I}}{2} \mathrm{P}+p\right) \cos \alpha \text { tang. } \psi & =\frac{70.247}{\mathrm{R}}=\frac{68 \mathrm{I} \cdot 382}{\mathrm{R}}
\end{aligned}
$$




$$
\begin{aligned}
\mu \lambda \mathrm{P} \cos . \alpha & =29.109 \\
(\mathrm{P}+\varepsilon \mathrm{P}+\pi) \text { fin. } \alpha & =482.634 \\
(\mathrm{P}+\pi) \cos . \alpha \text { tang. } \omega & =124.282 \\
\mathrm{R}^{\prime} & =636.025
\end{aligned}
$$

Three horfes, therefore, whofe ftrength is $\mathbf{M}=1200$ lib. and their velocity on this kind of road $G=$ to feet, could draw the burden $P=1800$ lib. on a four-wheeled carriage at the rate of $g=2.47$ feet per fecond, and on a two-wheeled carriage about $g^{\prime}=2 \cdot \eta^{2}$ feet.

\section{Section 24.}

On a horizontal road of the above nature we thall have for the fame carriages and load $R \quad \cdots 206.08$ and $R^{\prime}=158.09$. For a horle therefore whofe ftrength $\mathrm{M}=400$ lib., and velocity on this horizontal road $\mathrm{G}=\mathrm{I}_{5}$ feet, we hall have $g=$ $4^{\circ} 23$ and $g^{\prime}=5.5^{8}$ feet.

\section{Third Division. \\ Of the Refiflance on foft and even Roads.}

\section{SeElion 25.}

If the furface of the road be compofed of foft fand, flate, earth, clay, dirt, \&c. the wheels will fink into it; and the depth to which they fink will be greater the fofter and more fluid the matter is of which the road confifts.

Now as the increafe of refiftance depends on the depth to which the wheels fink, and as this depth depends on the nature of the matter, that is to fay, its hydroftatic power, which can be determined only by experiment, I fhall affume that a prifmatic body the thicknefs of which is $d d$ fquare inches and its weight 9 lib. finks merely by its gravity $c$ inches deep in the fluid matter, or difplaces $d d c$ cubic inches of it before it comes into equilibrimm : this matter, then, in regard to hydroftatic power produces the fame effeet as would be produced by an aqueous thid a cubic foot of which weighs $\frac{12^{3} q}{d d c}$ lib.

A load Q, PI. II. fig. 5. will prefs down both wheels on the axis $O$ till they have difplaced $\frac{d d c Q}{q}$ cubic inches of the fluid matter. If FR be the depth to which the wheels fink, and $\pi^{\prime}$ the circumference of a circle whofe diameter $=I$, the fuperficial content of the funken fegment GFG'R $=\mathrm{GO}^{2}\left(\frac{\pi^{\prime} \cdot 4 \mathrm{GOR}}{180^{\circ}}-\frac{1}{2} \mathrm{fin} . \mathrm{GOG}^{\prime}\right)$. And if the fellies 
be $b$ inches broad, we thall have for both wheels:

Hence we find,

$$
2 b \mathrm{GO}^{2}\left(\frac{\pi^{\prime} \cdot 4 \mathrm{GOR}}{180^{\circ}}-\frac{x}{2} \text { fin. GOG }\right)=\frac{d d c Q}{q} \text {. }
$$

$$
\frac{\pi^{\prime} \cdot \mathrm{GOR}}{90^{\circ}}-\mathrm{fin} . \mathrm{GOG}^{\prime}=\frac{d d \cdot \mathrm{Q}}{b q \cdot \mathrm{GO}^{2}} ;
$$

from which equation the angle GOR neceffary for determining the refiftance is found.

\section{Four-wheeled Carriages.}

\section{Section 26.}

As the wheels in this cafe have funk to the points $G, G^{\prime}$ of their circumference, there arifes in the point $G$ an impediment to the motion, which will be greater or lefs according as the furface of the road is covered with more or lefs fluid matter; that is, according to the greater or lefs extent of the angle which the fides of as fteep an accumlation as poffible of this matter forms with the level furface. This angle, which gives the moft convenient meafure of the fluidity, I fhall in future call $\beta$.

Now if GFG' were an excavation in a perfectly folid road, $G$ would oppofe the moving power $O V$ in confequence of the power of gravity $\mathrm{OQ}=\mathrm{S}$ with a power $\mathrm{OR}=\mathrm{S} \cos$. $\alpha$, the momentum of which is OR.GR $=\mathrm{S} \cos$, $\alpha$. GO fin. GOR. If the part of the moving power employed for this purpofe be K, its momentum K.GU $=$ K.GO cos. GOR ; fo that $\mathrm{K}=\mathrm{S}$ cos. $\alpha$ tang. GOR. If the angle for the forewheels be $G O R=\xi$, and for the hind-wheels $\mathrm{GOR}=n$, the refitance for the former $=\left(\frac{1}{2} P+p\right) \cos . \alpha$. tang. $\xi$, and for the latter $=\left({ }^{1} P+p^{\prime}\right)$ cos: $\alpha$ tang $n$; confequently, the increafe of refiftance for the four-wheeled carriages $=$ cos. $\alpha\left[()^{ \pm}-\mathrm{P}+p\right)$ tang. $\xi+\left(\frac{i}{2} \mathrm{P}+p^{\prime}\right)$ tang. $\left.n\right]$. With this power $G$ oppofes a refiftance as a fixed point, but, as a fluid, it yields and oppofes a lefs power the greater its fluidity, the coefficient of which is fin. $\beta$. The increafe of refiftance on roads of this kind is therefore $=\cos . \alpha$ fin. $\beta\left[\left(\frac{x}{2} \mathrm{P}+p\right)\right.$ tang. $\xi+\left(\frac{1}{2} \mathrm{P}+p^{\circ}\right)$ tang. $\left.n\right]$; confequently the whole refiftance for four-wheeled carriages:

$$
\begin{aligned}
& \mathrm{R}=\frac{x}{2}(m+n) \times \mathrm{P} \cos . \alpha+\left(\mathrm{P}+p+p^{\prime}\right) \text { fin. } \alpha \\
& \left.+\cos . \alpha \text { fin. } \beta\left[(\mathrm{P}+p) \text { tang. } \xi+\left({ }_{i}^{2} \cdot \mathrm{P}+p\right) \text { tang. n. }\right]\right\}
\end{aligned}
$$

\section{Two-wbeled Carriages.}

\section{SeEtion 27.}

If we make the angle GOR $=9$, it will be found in the Vox. XIII. No. 51 . 
fame manner as in the preceding fection, that the increafe of refitance on roads of this clals is $=(P+\pi) \cos . \alpha$ fin. $\beta$ tang. 9 ; and the whole refiftance for two-wheeled carriages will be,

$$
\left.\begin{array}{rl}
\mathbf{R}^{\prime}= & \mu \lambda \mathrm{P} \cos . \alpha+(\mathrm{P}+\varepsilon \mathrm{P}+\pi) \text { fin. } \alpha \\
& +(\mathrm{P}+\pi) \cos . \alpha, \text { fin. } \pi, \text { tang. } 9 .
\end{array}\right\}
$$

1II. Obfervations on thefe Formula $R$ and $R^{\prime}$.

\section{Section 28.}

Thefe expreffions for $R$ and $R^{\prime}$ as above found contain, as we thall foon fee, a complete folution of the problem, as they anfwer for all the three claffes of roads.

Ift, For folid and even roads where $\xi=0, n=0,9=0$, and $\beta=90^{\circ}$, they give for $R$ and $R^{\prime}$ the fame values as thofe found for refittance in the firft divifion.

$2 \mathrm{~d}$, For folid and uneven roads where $\xi=\varphi, n=\psi, 9$ $=\omega$, and $\beta=90^{\circ}$, we have the fame values for $R$ and $\mathbf{R}^{\prime}$ as thofe found in the fecond divifion.

3 d, For roads with ftanding water on a folid bottom (which, however, muft not reach to the fpokes, becaufe in this cafe the refiftance muft be determined in another manner), where $\beta=0$, we thall have $R$ and $R^{\prime}$ as in the firft divifion. In this cafe, no perceptible increafe of refiftance arifes: on the other hand, on fuch roads $\mathbf{G}$ and confequently $V$ is lefs than on roads of the firtt clafs.

4 th, When one of the angles $\xi, n$, or $\vartheta$, is a right angle; that is, when $P$ is fo great that the wheels fink up to the axle in the foft furface, the refiftance becomes infinitely great, except in that cafe only where the fluid is very thin, and $\beta=0$; in which cale inftead of an infinitely great refiftance we thall have only an indefinite increafe $=0 . \infty$, which cannot be determined from the grounds mentioned in the third obfervation.

In the laft place, it is to be obferved in regard to thefe exprefions,

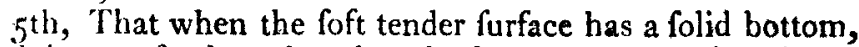
and is not fo deep that the wheels can penetrate into it according to the laws of hydroftatics, the angle GOR muft not be determined according to the abfolute finking, but according to the depth of the folid bottom.

6th, That for lefs fluid matters, fuch as moift fand, moift earth, ftiff clay, dirt, \&c. in the expreffion for the refiftance of four-wheeled carriages, $R$ the laft member for the hindwheels $\left(\frac{x}{2} P+p^{\prime}\right)$ cos. $\alpha$, fin. $\beta$, tang. $n$, vanifhes, becaufe thefe wheels run in the ruts formed by the fore-wheels. 
IV. Comparifon between the two Kinds of Carriages.

Seczion 29.

On roads of this clafs, four-wheeled carriages are to be preferred to two-wheeled, when one of the five following conditions takes place:

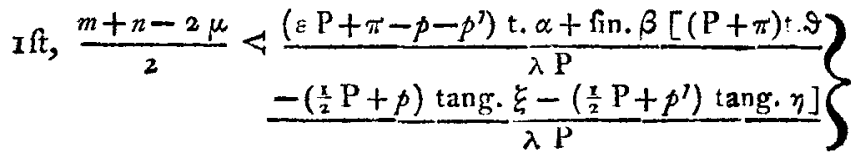

$$
\begin{aligned}
& \left.2 \mathrm{~d}, p+p-\pi<\varepsilon \mathrm{P}-\frac{(m+n-2 \mu) \frac{1}{2} \lambda \mathrm{P}+\text { fin. } \beta[(\mathrm{P}+\pi) \text { t. } .9}{\text { tang. } \alpha}\right] \\
& \left.\frac{\left.-\left(\frac{x}{2} P+p\right) \text { tang. } \frac{\xi}{\xi}-\left(\frac{1}{2} P+p^{\prime}\right) \text { tang. } \eta\right]}{\text { tang. } \alpha}\right\}
\end{aligned}
$$

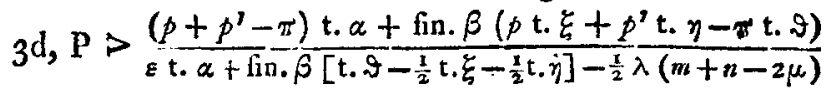

$$
\begin{aligned}
& \text { 4th, } \left.\varepsilon>\frac{(m+n-2 \mu) \frac{1}{2} \lambda \mathrm{P}+\left(p+p^{\prime}-\pi\right) \text { tang. } \alpha+\text { fin. } \beta}{\mathrm{P} \text { tang. } \alpha}\right)
\end{aligned}
$$

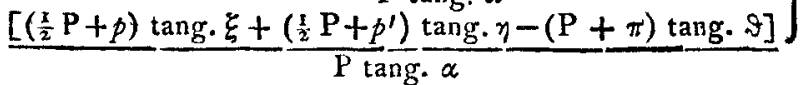

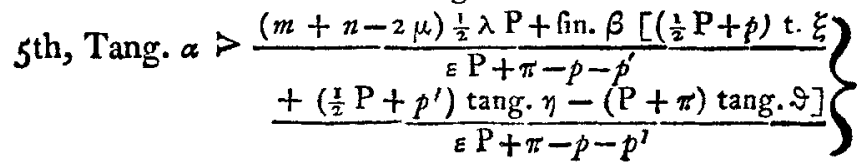

That thefe five conditions are general, and contain the conditions found for the two claffes before treated, fection 16 and fection 17 , it is fuperfluous to mention, after what has been faid in the firft two remarks of the preceding fection.

Application to fome determinate Cafes.

EXAMPLE I.

\section{Section 30 .}

I poured very dry coarfe red fand, Pl. II. fig. 6. after I had found the angle $\beta=30^{\circ} .4^{2}$ (that is to fay, $\mathrm{AB}=16$ inches and $\mathrm{CD}=4_{4}^{3}$ inches), into a veffel, and, fmoothing the furface of it after each experiment by thaking it fo as to obtain the fame degree of foftnefs, I took a fteel rod weighing 5 lib., which I had caufed to be conftructed for magnetic experiments, and which was juft an inch fquare, and pufhed it feveral times into the fand in a gentle manner, to prevent the acquired velocity from making it fink deeper than the equilibrium fought for. Sixteen experiments of this kind, in which the greateft difference did not amount to half a line, 


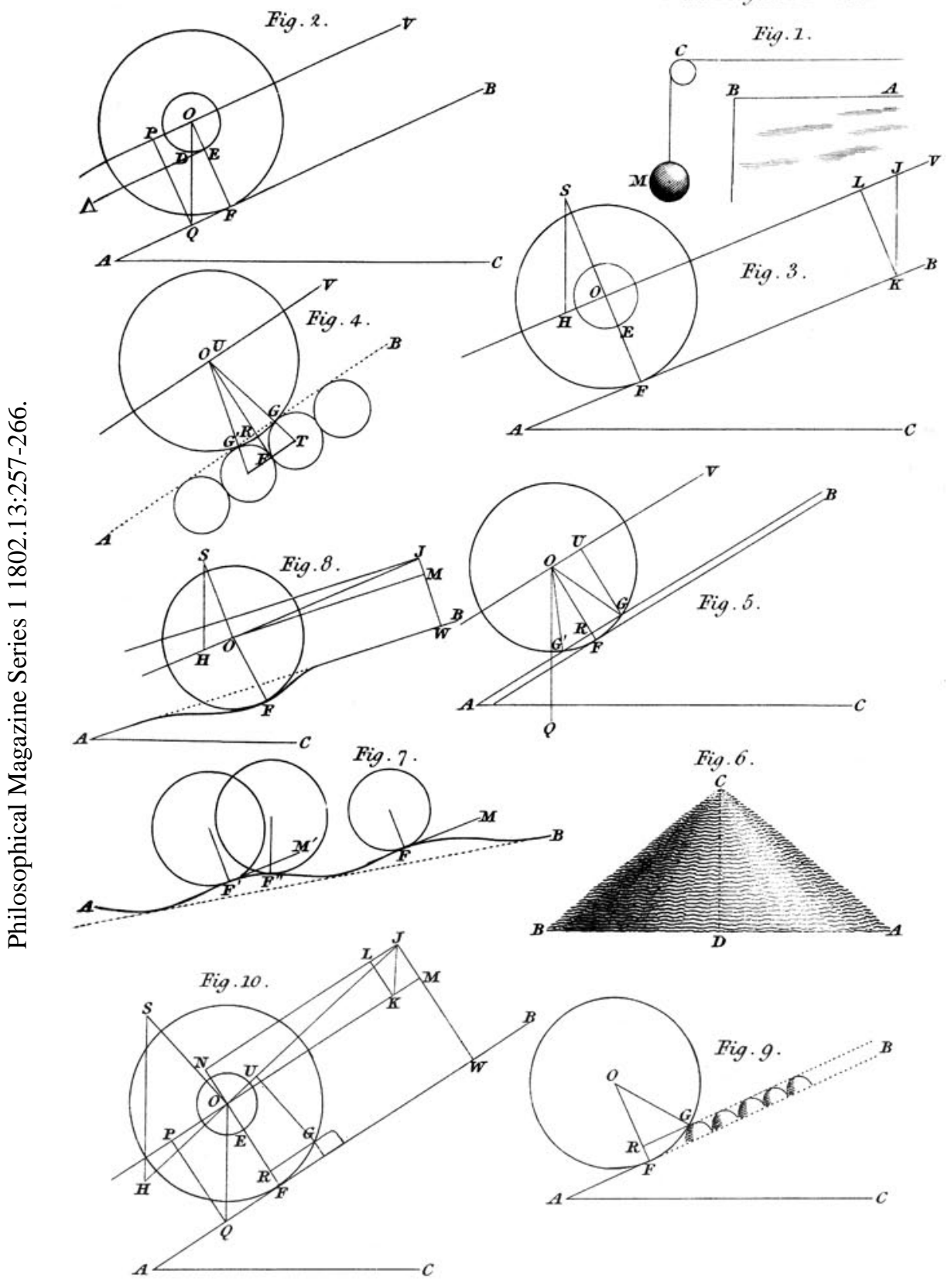

Lowry sculp. 
gave as a medium of the finking $\frac{7}{27}$ inches; fo that $d d c=\frac{7}{24}$ inches, and $q=5 \mathrm{lib}$.

Let the road confift of fuch foft fand; let the inclination $\alpha=4^{\circ}$; the load $P=1200$ lib., the weight of the wheels $p=84$ lib. $p^{\prime}=110$ lib., $\pi=132$ lib.; their diameter in the fame order as before, $3 \mathrm{I}_{\frac{1}{2}}^{\frac{1}{2}}, 42$, and 49 inches. Alfo let $m=\frac{1}{9}, n=\frac{1}{12}, \mu=\frac{1}{14}, \lambda=\frac{1}{5} ;$ OS $=2 \frac{6}{7}$ feet and OI $=$ I 2 feet ; fo that $\varepsilon=\frac{1}{60}$. In the laft place, let $b=3$ inches, confequently,

$$
\begin{aligned}
& \frac{\pi \xi}{90^{\circ}}-\text { fin. } 2 \xi=0.053^{61} \text {, and } \xi=19^{\circ} 47^{\prime} \\
& \frac{\pi^{\prime} \eta}{90^{\circ}}-\text { fin. } 2 n=0.03130 \text {, and } n=16^{\circ} 30^{\prime} \\
& \frac{\pi^{\prime} \cdot 9}{90^{\circ}}-\text { fin. } 2 \vartheta=0.04315 \text {, and } \vartheta=18^{\circ} 23^{\prime} \\
& \frac{1}{2}(m+n) \lambda \mathrm{P} \cos . \alpha=23.276 \\
& \left(\mathrm{P}+p+p^{\prime}\right) \text { fin. } \alpha \quad=97.242 \\
& \left(\frac{1}{2} \mathrm{P}+p\right) \text { cos. } \alpha \text {, fin. } \beta \text {, tang. } \xi=125.3 \circ 3 \\
& \left(\frac{1}{2} \mathrm{P}+p^{\prime}\right) \cos . \alpha \text {, fin. } \beta \text {, tang. } n={ }_{107} \cdot 112 \\
& \mu \lambda P \cos . \alpha \\
& (\mathrm{P}+\varepsilon \mathrm{P}+\pi) \text { fin. } \alpha \\
& (P+\pi) \cos . \alpha \text {, fin. } \beta \text {, tang. } 9 \\
& \mathrm{R}=352 \cdot 933 \\
& =17 \cdot 10 \mathrm{I} \\
& =94^{\circ} 31 \mathrm{I} \\
& =225^{\circ} 45^{\circ} \\
& 336 \cdot 862
\end{aligned}
$$

If we fuppofe for two horfes $M=800$ and $\mathbf{G}=\mathbf{I}$ feet, we thall have $g=3.696$ feet and $g^{\prime}=3.851$ feet in a fecond.

However well the two-wheeled carriages may be conftrueted, however fmall the burden, the inclination of the road, and the height of the centre of gravity, the refiftance is only a little lefs than for four-wheeled carriages. To fhow how much depends on the proportion of the wheels, and particularly in regard to carriages of this kind, I thall in the following examples change only the wheels of the twowheeled carriages.

\section{EXAm PLE II. \\ Section $3 \mathrm{r}$.}

Let every thing be as before, only for the two-wheeled carriages let $\mathrm{GO}=\mathrm{I} 6$ inches, $\pi=85 \mathrm{lib}$., and $\mu=\frac{7}{64}$; therefore $9=24^{\circ} 15^{\prime}$, and

$$
\begin{array}{ll}
\mu \times P \text { cos. } \alpha & =26.186 \\
(P+\varepsilon P+\pi) \text { fin. } \alpha & =91.032 \\
(P+\pi) \cos \alpha, \alpha \text { fin. } \beta, \text { tang. } s= & =294.808 \\
412.026
\end{array}
$$


Therefore we thall have as before $R=35^{\circ} 933$ lib., but $\mathrm{R}^{\prime}=412.026 \mathrm{lib}$; confequently $g=3.696$ feet, and $g^{\prime}=$ $3^{\cdot 102}$ feet.

\section{EXAMPLE III.}

\section{Section 32 .}

After a feries of fimilar experiments with fomewhat coarfer and very moift red fand, I found $\beta=43^{\circ} r 9^{\prime}$, and $c=\frac{5}{T^{2}}$ inch at $\mathbf{a}$ medium. But it is to be obferved that the impreffions made by immerfing the fteel rod did not difappear as from the above dry fand, but remained after it was drawn out; and therefore after each experiment I was obliged to make the fand even and foft by thaking it, and to render its furface fmooth by a llight preffure proportioned to the exact meafurement of the depth; by which means it loft a little of its natural foftnefs. When I placed the rod again in the remaining impreffion it did not become deeper. Hence follows what we are tanght by experience in general in regard to moift fand, moift earth, thick mud, \&rc. that the hind-wheels when they revolve in the ruts formed by the fore-wheels do not make them deeper, and confequently experience no refiftance from finking down; fo that in the value of $R$, fection 26, found for four-wheeled carriages, in fuch cafes the laft member $\left(\frac{1}{2} \mathrm{P}+p\right)$ cos. $\alpha$, fin. $\beta$, tang. $n$ vanifhes.

Let us fuppofe then, as in the firft example, fection 30 , that the angle $\alpha=4^{\circ}$ the weight of the wheels, $p=84$ lib., $p^{\prime}=110$ lib., $\pi=13^{2}$ lib.; and their femidiameter in the fame order, $\mathrm{GO}=15 \frac{3}{4}, 21,24 \frac{1}{2}$ inches, and $b=3$ inches ; alfo let $m=\frac{1}{9}, n=\frac{1}{\frac{1}{2}}, \mu=\frac{1}{4}, \lambda=\frac{1}{5}, \varepsilon=\frac{1}{6}$, and $g=$ 5 lib. If the load be $\mathrm{P}=900$ lib., and $d d c=\frac{5}{x_{2}}$ cubic inches, we fhall have

$$
\begin{aligned}
& \frac{\pi^{\prime} \xi}{90^{\circ}}-\text { fin. } 2 \xi=0.05980 \text {, and } \xi=20^{\circ} 32^{\prime} \\
& \frac{\pi^{\prime} \eta}{90^{\circ}}-\text { fin. } 2 n=0.03527 \text {, and } n=17^{\circ} 10^{\prime} \\
& \frac{\pi^{\prime} 9}{90^{\circ}}-\text { fin. } 29=0.04776 \text {, and } S=19^{\circ} 10^{\prime} \\
& \frac{x}{2}(m+n) \lambda \mathrm{P} \cos . \alpha=17457 \\
& \left(\mathrm{P}+p+p^{\prime}\right) \text { fin. } a \quad=76.313 \\
& \left(\frac{1}{2} \mathrm{P}+p\right) \cos . \alpha \text {, fin. } \beta \text {, tang. } \xi=136.878 \\
& \mathrm{R}=\overline{2,30 \cdot 648} \\
& \mu \lambda \mathrm{P} \cos . \alpha=\mathbf{1 2} .826 \\
& (\mathrm{P}+\varepsilon \mathrm{P}+\pi) \text { fin. } \propto \text { 列 } \\
& (P+\pi) \text { cos. } \alpha \text {, fin. } \beta \text {, tang. } 9=243.414 \\
& \text { S } 3 \\
& R^{\prime}=\overline{3^{2} 9^{2} 275}
\end{aligned}
$$


If for a horfe on this road $M=400$ lib. and $G=12^{\text {I }}$ feet, we hall have $g=3.012$ and $g^{\prime}=1.162$ feet in a fecond.

Allo if the hind-wheels do not run exactly in the ruts formed by the fore-wheels, and if the member $\left({ }^{1} P+p\right.$ ) cos. $\alpha$, fin. $\beta$, tang. $n=118.389$, cannot be entirely omitted, we fhall fitll have $R<R^{\prime}$ even when only the fourth part of it vanifhes.

[To be continued.]

XLIV. Some Account of EDWa RD Jenner, M. D.

\section{$\mathrm{T}$}

H IS gentleman, who has diftinguifhed himfelf fo much in the annals of medicine by bringing forward the vaccine inoculation to public notice, and who has been thought worthy of national remuneration on that account, is a fon of the Rev. Stephen Jenner, formerly vicar of Berkley in Gloucefterfhire, a man highly refpected by all thofe who had the pleafure of his acquaintance. Edward was born about the yeat 1749 , and received his education at Cirencefter in the fame county. Having made a confiderable progrefs in claffical learning, and howing an early attachment to the ftudy of phyfic, he was placed under the care of Mr. Ludlow, an eminent furgeon at Sodbury, a large market-town between Brittol and Wotton under Edge. After remaining with this gentleman fome time, during which be applied with affiduity, and made rapid improvement, he repaired to London to complete his medical education, and became a houfe-pupil to the celebrated John Hunter. In this fituation he continued two years, and availed himfelf with great fuccefs of the inftruction of fo able a mafter. At the expiration of this period he removed to berkley, where he commenced practice, and met with confiderable encouragement. In the mean time he ftill kept up his intimacy with $\mathrm{Mr}$. Hunter by a regular correfpondence; and the frequent mention which Mr. Hunter has made of him in his works is a ftriking inftance of the favourable opinion which he entertained of his abilities. An ingenious paper on the natural hittory of the cuckow, in a letter addreffed to Mr. Hunter, was communicated by him to the Royal Society, and was inferter in the Philofophical Tranfactions for 1788 . Several other papers of his on intricate fubjects in natural hiftory were publifhed about the fame time. In $\mathrm{r}_{778}$ he married mifs Catherine Kingfcote of Kingfcote in Gloucefter(hire, by whom 